\title{
Attendance at accident and emergency for deliberate self harm predicts increased risk of suicide, especially in women
}

Cooper J, Kapur N, Webb R, et al. Suicide after deliberate self-harm: a 4-year cohort study. Am J Psychiatry 2005;162:297-303.

\section{How prevalent is suicide in people who have committed deliberate self harm?}

\section{METHODS}

$-$

Design: Prospective cohort study.

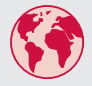

Setting: Four accident and emergency departments in

Manchester, UK; 1997 to 2001

으 Population: 7968 people (median age of 30 years) attending September 1997 and August 2001.

Prognostic factors: Attendance at accident and emergency for deliberate self harm.

3

Outcome: Suicide rates. Deaths by suicide were identified using

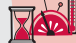

the National Confidential Inquiry Into Suicide and Homicide by People With Mental Illness database of the Office of National Statistics. Confirmed suicides and deaths from unknown cause (ICD-9 codes) were considered suicides. Suicide rates in the study population were compared with those for general population of Manchester to give standardised mortality ratios (SMRs)

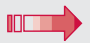

Follow up period: Four years.

\section{MAIN RESULTS}

Between September 1997 and August 2001, the suicide rate was 371 per 100000 in people who had attended accident and emergency because of deliberate self harm. Suicide rates were greatest within the first six months of the self harm episode (561.6 per 100000 ). Overall, the risk of suicide was 15 times higher in people who had self harmed than for the general population of the region (SMR 15.4, 95\% CI 11.8 to 19.9). The risk of suicide in women who had self harmed was 23 times higher than for women in the general population of the region (SMR 23.2, 95\% CI 14.5 to 35.1 ). The risk of suicide in men who had self harmed was 13 times higher than for men in the general population of the region (SMR 12.9, 95\% CI 9.2 to 17.8).

\section{CONCLUSIONS}

People attending accident and emergency because of deliberate self harm have a high risk of suicide. Suicide rates are highest within the

For correspondence: Dr J Cooper, Centre for Suicide Prevention, University of Manchester, 7th Floor, Williamson Building, Oxford Road, Manchester M13 9PL, UK

Sources of funding: Manchester Health Authority, South Manchester University Hospitals, National Health Service (NHS) Trust, Manchester Healthcare NHS Trust, North Manchester Healthcare NHS Trust, and Mental Health Service of NHS Trust. first six months of the self harm episode, and the risk of suicide relative to the general population is greater in women who present with self harm than in men.

\section{Commentary}

7 his well conducted prospective four year follow up of deliberate self harm (attempted suicide) is one of the largest of its kind, nearly matching a Scottish cohort of 8300 suicide attempters followed for 13 years, also linked to a mortality dataset.' The profile of risk factors developed by Cooper et al should alert emergency room staff to people with deliberate self harm (DSH) at particular risk for suicide. Risk factors include not living with a close relative, endeavouring to avoid discovery of the DSH and current abuse of alcohol, which carries a two- to threefold suicide hazard. Self cutting (self mutilation), previous psychiatric treatment, and the presence of physical health problems also emerged as risk factors. Standardised mortality ratios for women attempting suicide (calculated against the population of England and Wales) much exceeded those for men.

None of these risk factors would be surprising to a clinician with experience of suicidal behaviour, except perhaps self cutting. Self mutilation is usually regarded as embodying almost no suicidal intent and typically begins in adolescence when the person discovers that anger, tension, or dissociative numbness may be temporarily relieved by cutting the body and drawing blood. However, one third of self mutilators also make serious suicide attempts at some time, just as many people who make serious suicide attempts may not proceed to suicide. ${ }^{2}$ Effort made to conceal the act of attempting suicide or DSH is an important risk factor incorporated into the Precautions subscale of the Beck Suicide Intent Scale. In this 5-10 year follow up of suicide attempters, the risk of subsequent suicide increased $67 \%$ with each additional point scored in the precautions subscale. ${ }^{3}$ The study also found a strong association of subsequent suicide with alcohol abuse as had Hall et al.

As half of suicides have a history of prior DSH, the findings by Cooper et al strengthen our knowledge of the warning signs. However, a profile of risk factors found by analysis with one cohort may not be replicated with a subsequent cohort and accurate prediction of suicide at the individual level so far eludes us. ${ }^{2}$ The present study requires replication, preferably by other centres, and the Manchester group have warned elsewhere against a "high risk approach" to the treatment of DSH (the majority of DSH repeaters came from those evaluated as low risk). ${ }^{4}$ Isaac Sakinofsky MD, FRCPC, FRCPsych

Centre for Addiction \& Mental Health and University of Toronto, Toronto, Canada

1 Hall DJ, O'Brien F, Stark C, et al. Thirteen-year follow-up of deliberate self harm, using linked data. Br J Psychiatry 1998;172:239-42.

2 Sakinofsky, I. Repetition of suicidal behaviour. In: Hawton K, Van Heeringen $\mathrm{K}$, eds. The international handbook of suicide and attempted suicide. Chichester: John Wiley \& Sons, 2000:385-404.

3 Beck AT, Steer RA. Clinical predictors of eventual suicide: a 5- to 10-year prospective study of suicide attempters. J Affect Dis 1989;17:203-9.

4 Kapur N, Cooper J, Rodway C, et al. Predicting the risk of repetition after self-harm: cohort study. BMJ 2005;330:394-5. 